\title{
Implementing continuous quality improvement in general practice: the whole package or a series of projects?
}

Continuous quality improvement is a complex approach to improvement that describes an all encompassing "package" of principles, methods, and techniques (box). For large organisations setting out to change working practices in line with the principles of continuous quality improvement, implementation of the complete package may be appropriate. But for much smaller organisations with fewer resources - such as general practices - such a task may seem overwhelming. Yet continuous quality improvement has been shown to work in general practice. ${ }^{12}$ The evidence suggests, however, that implementing it gradually, starting with small scale projects, may be more successful in general practice, and a quality improvement report in this issue adds further support for this approach. ${ }^{3}$

- Management activities, including leadership, a mission and shared vision, specific and realistic targets, enough resources, favourable changes in the organisation (quality meetings)

- Activities and planning based on factual data, such as performance measures, analysis of the organisation, patient satisfaction

- Improvement through a systematic approach, which includes planned activities (improvement projects), use of the quality cycle in all activities, use of specific tools and techniques, learning by experience

- Collaboration, which means that everyone is involved in quality management, a positive attitude/culture towards continuous quality improvement, team building, and (last but not least) patient participation

\section{Box 1 A package for continuous quality improvement}

Doctors often find it difficult to cope with continuous quality improvement when it is introduced as an all encompassing "package". Experiments with implementing continuous quality improvement in this way have shown that implementing and sustaining the approach is difficult. ${ }^{12}$ The obstacles include complaints from practice staff that they do not have enough time to implement the methods; they are worried that the methods make practice more bureaucratic, and they simply find it difficult to get the whole approach off the ground. ${ }^{2}{ }^{4}$ It has been suggested that these difficulties may be related to doctors' fear of losing their professional autonomy in direct patient care. ${ }^{5}$ Gustafson has suggested that the model of continuous quality improvement, and particularly its means of implementation, need to be adapted to the practice's existing level of performance. ${ }^{6}$ As doctors have a special interest in, and gain pride from, improving direct patient care, this suggests introducing continuous quality improvement through projects linked to improving direct patient care. ${ }^{7}$

In this issue of Quality in Health Care, Cox et al report a project where they improved the process of repeat prescribing in a busy general practice in Bournemouth, UK using continuous quality improvement methods ( $\mathrm{p} 119)$. $^{3}$
They emphasise that continuous quality improvement is not a target in itself, but a tool, a technique to help organisations in improving quality. Their report illustrates how a project based approach to quality improvement can work effectively in general practice. It also shows that doing small scale improvement projects, using the methods of continuous quality improvement, can have positive effects beyond the project itself. In this example, the staff taking part in the project not only showed greater interest in performance measures but also became aware that quality improvement can only really be achieved when patients (and other "customers") are also involved in the improvement process. In short, doing small scale improvement projects can produce changes that encourage staff to adopt attitudes needed for continuous quality improvement.

The message that can be derived from the article by Cox et al is that it is necessary to adapt not only the method of continuous quality improvement to the level of performance of the organisation but also the implementation strategy. For general practice, this implies a project based approach. Nevertheless, it is important that the first improvement project is successful. A first improvement project must be chosen carefully therefore. It needs to be truly small scale, to have a realistic chance of being achievable, yet the targets of the project also need to be challenging and deal with a real issue. Effective leadership and learning by experience are key for meeting these conditions. ${ }^{8}$ Most practices will therefore need facilitation by experts from outside as well as people who are responsible for the projects from the inside - as the Bournemouth project makes clear. ${ }^{3}$

Thus getting practices used to the principles of continuous quality improvement through running guided improvement projects is more likely in the long term to change attitudes and approach than attempts to introduce continuous quality improvement as a complete package. And through this process, of course, the implementation of continuous quality improvement itself is one of the most challenging improvement projects a practice can run.

HARRIE GEBOERS

GP and research fellow, Centre for Quality of Care Research, University of Nijmegen, the Netherlands

1 Geboers H, Grol R, van den Bosch W, et al. A model for continuous quality improvement in small scale practices. Quality in Health Care 1999;8:43-9. 2 Lawrence M, Packwood T. Adapting total quality management for general practice: evaluation of a programme. Quality in Health Care 1996;5:151-8. 3 Cox S, Wilcock P, Young J. Improving the repeat prescribing process in a busy general practice. A study using continuous quality improvement methodology. Quality in Health Care 1999;8:119-25.

4 Berwick D, Enthoven A, Bunker J. Quality management in the NHS; the doctor's role. BMF 1992;304:304-8.

5 Batalden P. Organizationwide quality improvement in health care. In: Al-Assaf A, Schmele A, editors. The textbook of quality in health care. Florida, Delray Bay: St Lucie Press, 1991:60-74.

6 Gustafson D. Lessons learned from an early attempt to implement CQI Principle. Qual Rev Bull 1992;18:333-9.

7 Geboers H, van der Horst M, Mokkink H, et al. Setting up improvement projects in small scale practices: feasibility of a model for CQI. Quality in Health Care 1999;8:36-43.

8 Berwick D. A primer on leading the improvement of systems. BMF 1996;312:619-22. 